\title{
Structural organization of emotional burnout in call-center operators with different communicative tolerance
}

\author{
Elena Suroedova $^{1^{*}}$ Li Tianqi ${ }^{2}$ \\ ${ }^{1}$ Don State University, 344000 Rostov-on-Don, Russia \\ 2 Jiangsu Normal University, 221009 Xuzhou, China
}

\begin{abstract}
Annotation. The author considers the problem of the state of the structure of emotional burnout in labor subjects with different levels of communicative tolerance. The study found that call center operators with an average level of tolerance are more pronounced manifestation of emotional burnout. The phases of emotional burnout are a basic component in the structure of emotional burnout.
\end{abstract}

Modern society is characterized by high variability and stress. Today, every day a person is faced with a variety of stress factors in everyday life, at work, even during rest. The high level of requirements to the competence of a specialist, the impact of the media on the psyche and consciousness, various complex life situations adversely affect the productivity and efficiency of professional activity. Organizational psychologists and HR specialists point to the link between the employee's involvement in a stressful environment and his health. Medical psychologists and occupational pathologists note that an employee who experiences constant negative effects of the environment develops psychosomatic diseases, depression, fatigue syndrome, symptoms of emotional and professional burnout, decreased performance are increasingly detected.

In Russia, despite the initiation by the government of the issue of the health of the nation and workers, not enough attention is paid to the introduction of health-saving technologies to enterprises. The same can be said about the education of a culture of work and rest in the subject of work: not every specialist has the knowledge and skills adequate, not to the detriment of health, stress and stress. Stress factors and the inability to adequately use the techniques of self-regulation of their emotional and mental state become the trigger for the development of emotional and professional burnout of the employee. Emotional burnout is understood as "a mechanism of psychological protection developed by a person in the form of complete or partial exclusion of emotions or reduction of their activity in response to psychotraumatic effects [2]. This leads to the fact that a person develops a stereotype of emotions, allowing not to waste energy resources. Being at the stage of distress, the subject of labor has violations in the communicative, emotional and volitional spheres, weakens health, psychosomatic symptoms appear.

\footnotetext{
${ }^{*}$ Corresponding author: suroedova@mail.ru
} 
At the end of the last century, researchers found that emotional disorders are more common among professionals professions man-man. Specialists begin to experience fatigue, emptiness, irritation and fatigue (teachers, salesmen, managers, nurses, doctors, etc.). In addition to the feeling of pain, professional efficiency decreases, competence deteriorates.

The profession of the call-center operator refers to the specialties where communication with the client, albeit indirect, takes a long time. Therefore, communicative tolerance is one of the leading competencies of the call-center operator. Usually it is defined as a system characteristic, a psychological ensemble, which presents different qualities of the individual, especially moral, characterological and intellectual [1]. The researchers note that it is in the ability to show respect, tolerance, leniency to customers in call-center operators is lost with the development of symptoms of emotional burnout.

Theoretical analysis of the literature has shown that today a large number of empirical studies are conducted on various aspects of the study of emotional burnout, but the problem is not yet solved and remains relevant. In modern studies, the external factors of emotional burnout of specialists of the profession such as "man-man" $[3,5,6]$. At the same time, it is not enough to study the psychological aspects of the work of call-center specialists, namely the relationship of communicative tolerance and emotional burnout of operators. Callcenter operator is a fairly new profession for Russia, which is now widespread in Russia. Operators are often students and unskilled professionals, without secondary special or higher education. In addition, communication between operators and customers is mediated, the operator can not get complete feedback, which often becomes a barrier to establishing an effective contact. HR managers and psychologists notice that this type of work often adversely affects the psyche of operators and leads to negative consequences, conflicts, health problems.

Objective: to identify the specific structure of emotional burnout in call center operators with different communicative tolerance.

The study involved 43 people, call center operators aged 18-42 years (25 men and 18 women).

Research methods: Diagnostic technique for general communicative tolerance developed by V.V.Boyko. Method for diagnosing the level of emotional burnout V.V. Boyko.

Mathematical data processing was carried out using the methods of descriptive statistics, comparative statistics (Mann-Whitney U-test), correlation analysis (r-Pearson), structural and psychological analysis by generalized indicators of organization, integrability and differentiation index (A.V. Karpov), $x^{2}$ method for comparing matrices and structuregrams for their "homogeneity-heterogeneity".

As a result of diagnostics by the method of V.V. Boyko, it was found that $49 \%$ of callcenter operators have a high level of communicative tolerance. Specialists with a high level of tolerance show respect and interest in the client, show flexibility and friendliness, tolerance for "complex" clients. $51 \%$ of the subjects showed an average level of communicative tolerance. In communication, call center operators with an average level of tolerance may be less sensitive to customer needs, be sharp in speaking, less tolerant of personal, characterological or physical characteristics of customers.

The following pattern is noteworthy: with an increase in the level of communicative intolerance, some indicators of phases and symptoms of emotional burnout increase. There are also differences in the severity of burnout among subjects with different levels of communicative tolerance.

Empirically, based on a comparative analysis, it was found that a higher level of symptom manifestation is Dissatisfaction with oneself $\left(\mathrm{U}=120.0, \mathrm{p}=0.007\right.$, with $\mathrm{n}_{1}=21$, $\left.\mathrm{n}_{2}=22\right)$, Emotional detachment $\left(\mathrm{U}=133.0, \mathrm{p}=0.017\right.$, with $\left.\mathrm{n}_{1}=21, \mathrm{n}_{2}=22\right)$, Personal 
detachment (depersonalization) $\left(\mathrm{U}=129.0, \mathrm{p}=0.013\right.$, with $\left.\mathrm{n}_{1}=21, \mathrm{n}_{2}=22\right)$, was found in the group of call center operators with an average level of tolerance than in a group with high values of communicative tolerance. For a subject with an average communicative level, problems and events occurring at work begin to worry less, attitudes appear that working with people is not interesting and does not represent social value; she ceases to give satisfaction.

According to the scale of emotional burnout, Psychosomatic and psycho-vegetative disorders $\left(\mathrm{U}=105.0, \mathrm{p}=0.002\right.$, with $\left.\mathrm{n}_{1}=21, \mathrm{n}_{2}=22\right)$, it was found that it is much more pronounced for call center operators with a high level of tolerance. A subject with a high level of tolerance is characterized by a manifestation of irritability, internal stress and fear, a state of increased fatigue, exhaustion, and depressive manifestations.

Based on the statistical analysis, it was found:

higher rates of emotional burnout were found in call-center operators with an average level of communicative competence;

higher rates of Psychosomatic and psycho-vegetative disorders symptom were found in call-center operators with a high level of communicative tolerance.

Analyzing the results and identifying statistically significant differences in the manifestation of emotional burnout among call center operators with different levels of communicative tolerance, a hypothesis was put forward that there are differences in the structural organization of components of emotional burnout between call center operators with different levels of communicative tolerance.

Before starting the analysis of the structural organization of emotional burnout, it is necessary to determine the relationship between the symptoms and phases of emotional burnout.

The results of the correlation analysis of the general sample showed that there are direct, positive relationships between communicative tolerance and symptoms and phases of emotional burnout. The results of the correlation analysis of the total sample are presented in table 1 .

Table 1. The results of the correlation analysis of the study of emotional burnout and communicative tolerance

\begin{tabular}{|c|c|c|c|c|c|c|}
\hline & DOS & EaMD & Resistance & ED & PD & Exhaustio \\
\hline $\mathrm{r}$ &, 578 &, 321 &, 339 &, 411 &, 589 &, 456 \\
\hline $\mathrm{p}$ &, 000 &, 043 &, 032 &, 008 &, 000 &, 003 \\
\hline
\end{tabular}

Legend. Burnout symptoms: DOS - Dissatisfaction with oneself, EaMD - Emotional and moral disorientation, ED - Emotional detachment, PD - Personal detachment (depersonalization)

From Table 1 we see that there are close relationships between communicative tolerance and symptoms: Self-dissatisfaction, Emotional detachment, Personal detachment (depersonalization) and the phase of Exhaustion; weak positive connections were found between communicative tolerance and the symptom of Emotional and moral disorientation and the Resistance phase.

The data obtained allow us to say that the more often call center operators misunderstand customers and colleagues, reject their individual characteristics, categorize people, reluctance to control unpleasant feelings towards a partner, desire to "re-educate", unwillingness to forgive mistakes, inability to adapt to the nature and habits of another person, the more pronounced in professional activity are dissatisfaction with their labor results, coldness in relationships and relationships odeystvii with colleagues and clients, and detachment is not included in the activities, reluctance to help customers, sometimes in conflict, can not resolve it constructively. 
Further research involves a description of the structural organization of emotional burnout among call center operators with a high and medium level of communicative tolerance. For this purpose, matrices of inter-correlation relationships of symptoms and phases of emotional burnout were compiled. Each of the matrices was calculated separately for the selected groups of subjects through the correlation analysis procedure using the Pearson r-test $(\mathrm{p}<0.05$ and $\mathrm{p}<0.01)$.

Then, structural diagrams were constructed of significantly correlating symptoms and phases of emotional burnout and structural indices were calculated - the structure coherence index (SCI), the structure divergence index (SDI) and the general structure organization (GOI) [4]. In the groups of subjects only positive relationships were established between the phases and symptoms of emotional burnout.

Structural diagrams of symptoms and phases of emotional burnout in groups of callcenter operators with a high and medium level of communicative tolerance are presented in Figures 1 and 2 .

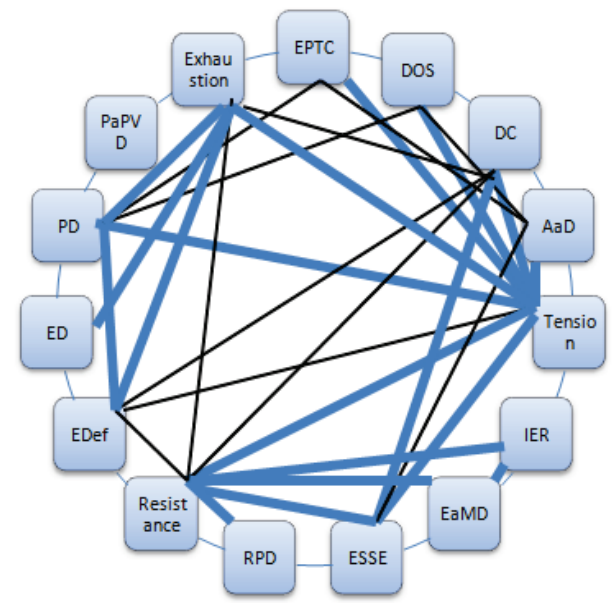

Fig. 1. Structural diagram of symptoms and phases of emotional burnout in call center operators with a high level of communicative tolerance

Legend. Burnout symptoms: EPTC - Experiencing psycho-traumatic circumstances, DOS Dissatisfaction with oneself, DC - "Depression into the cell", AaD - Anxiety and depression, IER Inadequate emotional response, EaMD - Emotional and moral disorientation, ESSE - Expanding the sphere of saving emotions, RPD - Reduction of professional duties, EDef - Emotional deficit, ED Emotional detachment, PD - Personal detachment (depersonalization), PaPVD - Psychosomatic and psycho-vegetative disorders. Burnout phases: Tension, resistance, exhaustion.

In both groups, the group (with an average and high level of communicative tolerance) revealed only positive connections between all symptoms and phases of emotional burnout.

Based on the presented data, the indices of the structural organization of emotional burnout for call center operators with a high and medium level of communicative tolerance were determined (Table 2). 


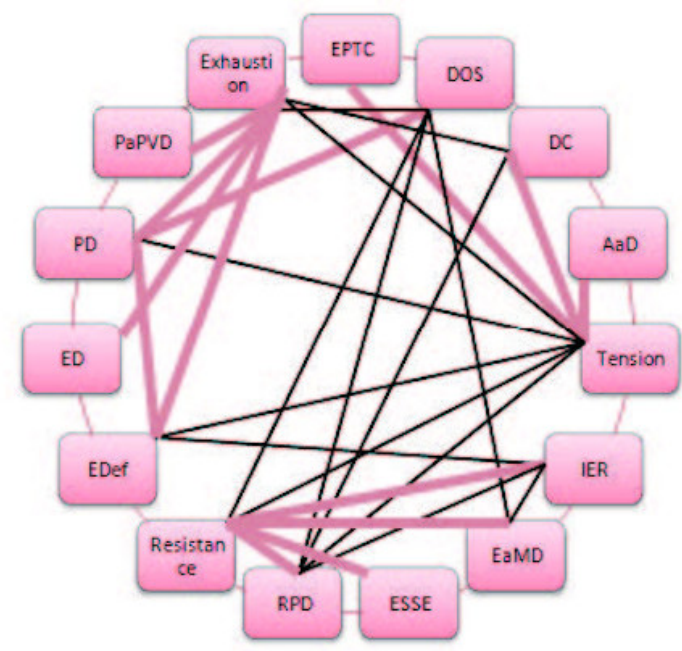

Fig. 2. Structural diagram of symptoms and phases of emotional burnout in call-center operators with an average level of communicative tolerance

Legend. Burnout symptoms: EPTC - Experiencing psycho-traumatic circumstances, DOS Dissatisfaction with oneself, DC - "Depression into the cell", AaD - Anxiety and depression, IER Inadequate emotional response, EaMD - Emotional and moral disorientation, ESSE - Expanding the sphere of saving emotions, RPD - Reduction of professional duties, EDef - Emotional deficit, ED Emotional detachment, PD - Personal detachment (depersonalization), PaPVD - Psychosomatic and psycho-vegetative disorders. Burnout phases: Tension, Resistance, Exhaustion.

Table 2 The values of the indices of the structural organization of emotional burnout in call center operators with different levels of communicative tolerance

\begin{tabular}{|l|l|l|}
\hline \multirow{2}{*}{ Indices } & \multicolumn{2}{|c|}{ Communicative Tolerance } \\
\cline { 2 - 3 } & High level & medium level \\
\hline Structure Coherence Index & 76 & 67 \\
\hline Structure Divergence Index & 0 & 0 \\
\hline General Organizability Index & 76 & 67 \\
\hline
\end{tabular}

Analysis of the data presented in table 2, allowed to draw the following conclusions:

1. A similar structure divergence index (IDS) was found in groups with different levels of communicative competence.

2. The degree of integration (coherence) of the structure (SCI) and the index of the general organization of the structure (GOI) of emotional burnout in a group with a high level of communicative tolerance are higher than in a group with an average level of tolerance.

To describe the qualitative uniqueness of the structures of emotional burnout among call center operators with a high and medium level of communicative tolerance, a comparative analysis of the content of the basic properties of the structure of emotional burnout for two groups was carried out. The data are presented in table 3 .

Table 3 Weighted values of symptoms and phases of emotional burnout in call center operators with a high and medium level of communicative tolerance

\begin{tabular}{|l|l|l|}
\hline \multirow{2}{*}{ Components } & \multicolumn{2}{|l|}{ Component weight } \\
\cline { 2 - 3 } & \multicolumn{2}{|l|}{ Communicative tolerance } \\
\cline { 2 - 3 } & High level $\left(\mathrm{W}_{1}\right)$ & medium level $\left(\mathrm{W}_{2}\right)$ \\
\hline Experiencing psycho-traumatic & 7 & 3 \\
\hline
\end{tabular}




\begin{tabular}{|l|l|l|}
\hline circumstances & & \\
\hline Dissatisfaction with oneself & 7 & 11 \\
\hline "Depression into the cell" & 12 & 7 \\
\hline Anxiety and depression & 9 & 3 \\
\hline Phases Tension & 26 & 19 \\
\hline Inadequate emotional response & 7 & 9 \\
\hline Emotional and moral disorientation & 9 & 7 \\
\hline Expanding the sphere of saving emotions & 11 & 3 \\
\hline Reduction of professional duties & 3 & 8 \\
\hline Phases resistance & 21 & 15 \\
\hline Emotional deficit & 15 & 10 \\
\hline Emotional detachment & 3 & 3 \\
\hline Personal detachment (depersonalization) & 13 & 11 \\
\hline Psychosomatic and psycho-vegetative & & \\
disorders & 0 & 3 \\
\hline Phases exhaustion & 16 & 18 \\
\hline
\end{tabular}

The data presented in Table 3 make it possible to detect the basic components of emotional burnout in call center operators with a high and medium level of communicative tolerance.

It should be noted that the basic component for a high and medium level of communicative competence is the phases of Stress $\left(\mathrm{W}_{1}=26, \mathrm{~W}_{2}=19\right)$, Resistance $\left(\mathrm{W}_{1}=\right.$ $\left.21, \mathrm{~W}_{2}=15\right)$ and Depletion $\left(\mathrm{W}_{1}=16, \mathrm{~W}_{2}=18\right)$. Of the symptoms of emotional burnout in the group with a high level of tolerance, the symptom of Emotional Deficiency $\left(\mathrm{W}_{1}=15\right)$ is distinguished.

Matrices of intercorrelation relationships calculated for the two groups were processed using the express- $\mathrm{x}^{2}$ method for their homogeneity-heterogeneity.

A comparison of the intercorrelation matrices with a high level and an average level of communicative competence $(\mathrm{p}=0.64)$ showed that the differences between the symptoms and phases of burnout are not statistically significant. Therefore, the compared structures of emotional burnout are heterogeneous, that is, qualitatively different in the weight of the elements in the compared structures.

Summarizing the data allows us to conclude that call center operators with a high level of communicative tolerance have a higher degree of structural organization of symptoms and phases of emotional burnout. It can be argued that the level of severity of communicative tolerance is interdependent with the degree of structure of emotional burnout. This indicates that the structure of emotional burnout among call center operators with a high level of communicative tolerance is more consistent than that of call center operators with an average level of communicative tolerance. This conclusion was also made on the basis of the fact that this study found that a higher level of emotional burnout is characteristic of call center operators with an average level of communicative tolerance.

\section{References}

1. V.V. Boyko, Psychoenergetics (Piter, St. Petersburg, 2008)

2. O.P. Gredyushko Bulletin of TSHPU. 2-3(17-18), 29-34 (2009)

3. L.V.Dimitrova, V.M.Dvurechenskaya, O.V.Mansurova, G.N. Uvarova, Meditsinskaya sestra. 1, 50-53 (2014)

4. A. A. Karpov General abilities in the structure of metacognitive personality traits (Yaroslavl State University, Yaroslavl, 2014)

5. E.A. Suroedova, N.N. Kazarnikova, Psychology of Education. 9, 35-41 (2018)

6. EA. Suroedova, Bulletin South Ural State University. 11, 97-105 (2018) 